ISAHP 1999, Kobe, Japan, August 12-14, 1999

\title{
Evaluation of a GA approach to AHP by Simulation
}

\author{
Noriyuki Matsuda, Nobuko Kato \& Satoshi Tamura \\ Inst. of Policy and Planning, Univ. of Tsukuba \\ Tennou-dai, Tsukuba, Ibaraki 305-8573, Japan \\ Dept. of Electronics, Tsukuba College of Technology \\ Amakubo 4-3-15, Tsukuba 305, Japan \\ Graduate School of Management Systems Sciences, Univ. of Tsukuba \\ 1-1-1 Tennou-dai, Tsukuba, Ibaraki 305-8573, Japan \\ mazda@shako.sk.tsukuba.ac.jp /nobuko@a. tsukuba-tech. ac. jp / k983208@dollar. keisei. tsukuba. ac. jp
}

\begin{abstract}
The viability of AHP seems to increase by incorporating GA to deal with ambiguities in ordinary human judgment. The purpose of this study is to evaluate Matsudats (1999) approach by simulation. Ambiguities inherent in ordinary judgment lead to indeterminacy about the final output of AHP and other decision-support tools. In this study, we first employed a wave model to compute the upper $(U)$ and lower $(L)$ boundaries for each judgment in AHP. Letting $R$ denote a response value, we obtained a triplet $\langle L, R, U\rangle$ for each AHP item from which numerous value combinations were possible. GA (Genetic Algorithm) helped us find a desirable combination of them in terms of $d p$ (degree of prudence) derived from the ratio of the highest priority adjusted for the number of alternatives. The initial generation was created under two coding schemes at each AHP level: biased and unbiased coding. We conducted a Monte Carlo simulation to test how well the present approach improved $d p$ from the original input. The test was part of usability evaluation of the present approach.
\end{abstract}

\section{Introduction}

AHP (Analytic Hierarchy Process), proposed by Saaty (1980), has been known to be quite a viable decision support tool in many areas. It derives relative priorities for a given alternative set from the weights accorded to hierarchically arranged decision items. Its user-friendliness stems from the localized evaluations within a hierarchy, since our natural judgments are mostly kanseic rather than rational. The contrast does not mean that kansei is irrational. Instead, they both constitute human intelligence, but differ in orientation as follows (Matsuda, 1997, 1999):

Rationality: Intelligent capacity oriented toward unambiguity, precision, rigor, consistency,... Kansei: Intelligent capacity that allows partial deviations from such standards.

In short, kansei enables us to live with practical efficiency when relevant information is partially available, optimal procedures are not completely known in the ever changing environments. Within reasonable bound, however, $k a n s e i$ leads us to attain, to a satisfactory extent, what rationality strives for.

Taking advantage of the hierarchical structure of AHP, its user can concentrate on localized or partitioned judgement, leaving systematic information integration to the tool. Nevertheless, we believe that the standard pairwise comparisons are too fragmented and repetitive for the user to maintain consistency even in the partitioned classes. As a solution, we suggest simultaneous multiple comparisons, making use of interactiveness of graphical computer interface (Matsuda, 1999).

To further enhance the practicality of AHP, we propose here an application of GA (Genetic Algorithms) in expectation of reaching a set of priorities among awfully many possibilities arising from 
ambiguities inherent in kanseic judgment. A procedural tool that bridges between GA and AHP is called a wave model that generates upper and lower bound values for each judged value of the user' $s$.

Ambiguous judgment is, in brief, assumedly measurable at least in intervals within which a response value falls. However, the partiality of kansei is likely to induce ambiguities in expressions about the boundaries., i.e., the second-order ambiguities. The recursive nature of such subjective ambiguities must be solved by a non-subjective procedure like our wave model to be explained in detail later. The model is expected to capture ambiguous judgment without imposing extra mental tasks on the users.

The present paper presents use of $\mathrm{GA}$ as a means to support kanseic evaluation of alternatives by AHP, employing the wave model as a means of ambiguity quantification. GA is expected to lead us to find a prominent alternative among numerous possibilities.

\section{Method}

The following explanations are based on the AHP structure shown in Figure 1 for illustrative purposes. Levels are numbered from 0 to 2 so that the user can add higher levels, if needed. Naming objects and classes constitute important part of programming in terms of information design (Matsuda, 1997).

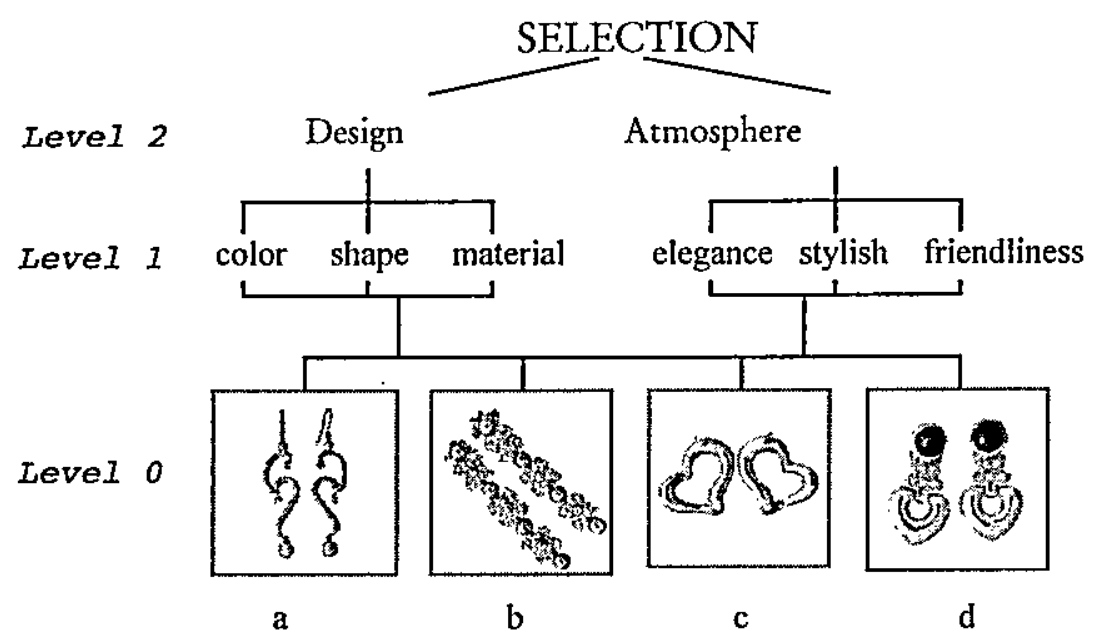

Figure 1. Evaluation structure by AHP

Wave model

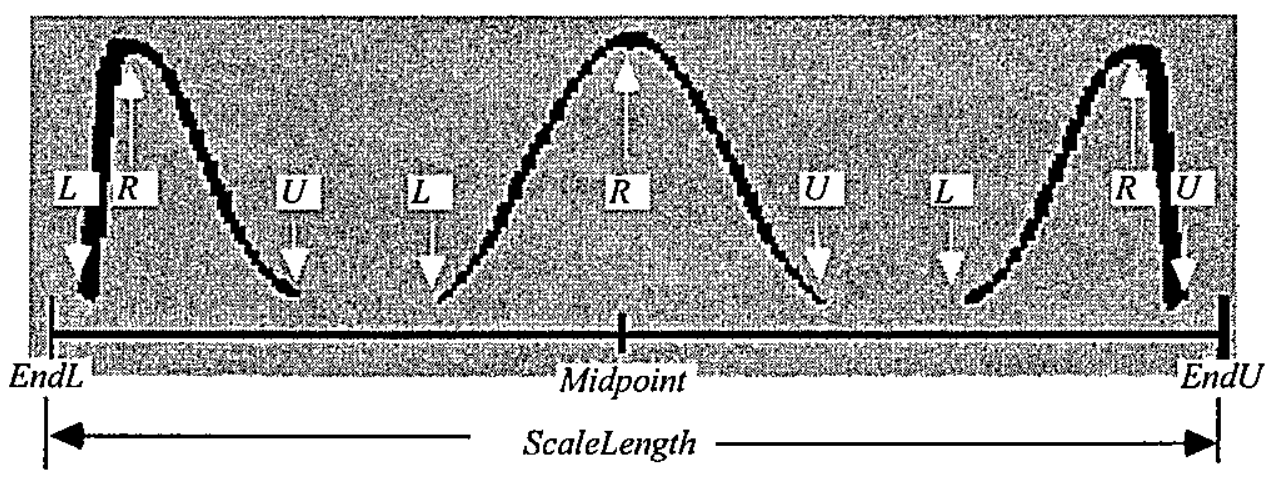

Figure 2. Sketches of the wave model 
The model is a metaphor of waves hitting wharves on both sides of a scale (Figure 2). The height of the distribution may represent the probability, the degree of confidence, or a fuzzy membership associated with a value on the scale. It was originally suggested by Gärdenfors, \& Sahlin (1983) as a conceptual device to deal with the subjective second-order probability. Though their idea is stimulating, they did not provide functional specifications to conduct the present work.

For the ease of presentation, we let $R, L$, and $U$ denote the response value, lower and upper ends of the associated interval, respectively. The distribution under the model is symmetrical when the response value $(R)$ falls on the middle of a scale, but becomes positively (or negatively) skewed toward the lower (or upper) end of the scale. Also, its range $(U-L)$ reaches maximum when $R$ is on the middle of the scale. The range decreases monotonously, as $R$ nears the scale ends. $\operatorname{Cos}()$ seems to be a natural choice for such function, being simple and mildly nonlinear. The argument of $\cos ()$ is the distance $(r e l D)$ of $R$ from the midpoint of a scale, Midpoint, relative to the half of the scale length, ScaleLength. The following are the formulas for computing $U$ and $L$ from $R$ :

$$
\begin{aligned}
& \text { if } R \leq \text { Midpoint } \\
& L=R \text {-(maxRange/2)* } \cos (\text { relD }) \\
& \text { otherwise } \\
& U=R+\left(\text { maxRange/2)* } \cos (r e l D)+a^{*} \sin (\text { relD })\right. \\
& L=R-\left\{(\text { maxRange/2 }) * \cos (\text { relDist })+a^{*} \sin (\text { relD })\right\} \\
& U=R+(\text { maxRange } / 2) * \cos (\text { relDist })
\end{aligned}
$$

where $a$ is the coefficient that determines the asymmetricity of the distribution. In the present work, maxRange and $a$ were set at 1.4 and 0.4 , respectively.

\section{Degree of prudence $(d p)$}

AHP priorities with small differences leave the user indecisive. If, in contrast, one or two alternatives are prudent in terms of priority, decision is easy. Under this premise, we introduce the following measure of prudence:

$$
d p=(\max (\{\text { priority }\})-1 / N) /(1-1 / N), \quad 0 \leq N \leq 1
$$

where $N$ is the number of alternatives. When all the priorities are equal it is 0 , whereas when only one alternative receives a positive. Note that AHP priorities are standardized so that they sum to 1 .

\section{Estimation by GA}

The task of GA is to find out the largest $d p$, by manipulating the combination of evaluations in terms of $\langle L, R, U\rangle$. The following explanation is based on the structure in Figure 1. A chromosome was created at each level to confine crossover within a level:

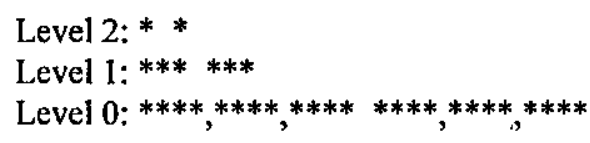

where * marks a locus. Items at Level 2 are separated by a space, which is used as a delimiter among the corresponding items or alternatives at lower levels. At level 0 , alternatives are to be arranged horizontally:

$$
\text { abcd,abcd,abcd abcd,abcd,abcd }
$$

The total number of assignment of $\langle L, R, U>$ to each locus amounts to 332 possibilities. 
The first generation consists of individuals whose genes are created by biased- and unbiased-coding. The former is favors particular alternatives, while the latter has no such favoritism.

Biased coding: The coding is unaffected by the judgment data differ at Level 0 , but rely on them at higher levels. At Level 0 , every alternative has its own gene in which $U$ is assigned to the corresponding loci , while $\mathrm{L}$ is assigned to the remaining loci (see Table 1 ).

Table 1. Biased coding at Level 0

\begin{tabular}{cc}
\hline Biased alternative & Coding \\
\hline $\mathrm{a}$ & ULLL,ULLL,ULLL ULLL,ULLL,ULLL \\
$\mathrm{b}$ & LULL,LULL,LULL LULL,LULL,LULL \\
$\mathrm{c}$ & LLUL,LLUL,LLUL LLUL,LLUL,LLUL \\
$\mathrm{d}$ & LLLU,LLLU,LLLU LLLU,LLLU,LLLU \\
\hline
\end{tabular}

Suppose that Table 2 shows the obtained (standardized) data at Level 0 . The Loci 1.22 and 2.2 at Levels 1 and 2, respectively, correspond to the largest value (.382) for $a$ at Level 0 .

Table 2. Indexed loci and hypothetical data at Level 0

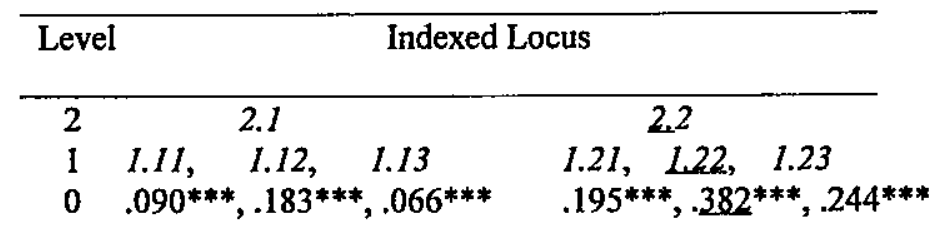

Note: ltalics are loci. Data of the other alternatives are marked by *.

Then, $U$ is assigned to these loci, while $L$ are assigned to the rest as follows:

Level 2: L U

Level 1: LLL LUL

Unbiased coding: This is divided into random coding in which an element of $\langle L, R, U\rangle$ is randomly chosen for every locus (see Table 3 ), and uniform coding in which all the loci at a given level share a single element. To exhaust the possibilities in the latter, three genes are created at each level as shown in Table 4.

Table 3. An example of random coding

\begin{tabular}{ccc}
\hline Level & \multicolumn{2}{c}{ Coding } \\
\hline 2 & L & R \\
1 & RRL & LRL \\
0 & LRLR, RRLL, LLLL & RRLR, LRLR,LURR \\
\hline
\end{tabular}


Table 4. Uniform coding

\begin{tabular}{ccc}
\hline Level & & Coding \\
\hline 2 & $\mathrm{~L}$ & $\mathrm{~L}$ \\
& $\mathrm{R}$ & $\mathrm{R}$ \\
& $\mathrm{U}$ & $\mathrm{U}$ \\
1 & LLL & LLL \\
& RRR & RRR \\
& UUU & UUU \\
0 & LLLL,LLLL,LLLL & LLLL,LLLL,LLLL \\
& RRRR,RRRR,RRRR & RRRR,RRRR,RRRR \\
& UUUU,UUUU,UUUU & UUUU,UUUU,UUUU
\end{tabular}

The chromosomes of an individual in the initial generation is created from union of the direct products of the above coding schemes:

$\{\text { random,uniform }\}_{\text {Level } 2} \otimes\{\text { random,uniform }\}_{\text {Level 1 }} \otimes\{\text { random,uniform }\}_{\text {Level } 0}$

$\mathrm{U}\{\text { biased }\}_{\text {Level } 2} \otimes\{\text { biased }\}_{\text {Level } 1} \otimes$ \{random,uniform,biased $\}_{\text {Level } 0}$

The 35 fittest individuals in terms of $d p$ are chosen as elite genes to remain the next generation. Additional individuals are created by crossover and mutation on the elite. Moreover, in order to avoid stagnation by incest, 20 individuals coded totaliy randomly were added. The same operation is repeated for the successive generations until no further improvement in $d p$ is expected.

\section{Results and concluding remarks}

To obtain baseline information, we first performed blind search using 1,000 individuals created by total ly random coding. The best $d p$ in 10 independent trials was only 0.157 . In contrast, the aforementioed GA started with only 400 individuals produced more than one elite genes with $d p$ above 0.200 only in three generations. Although the $20 \%$ improvement is not highly striking, efficiencies in time and in the size of individuals involved are certainly appreciable. More reusits will be reported at the conference.

\section{References}

Gärdenfors, P., \& Sahlin, N-E. (1983). Decision making with unreliable probabilites. British Journal of Mathematical and Statistical Psychology, 36, 240-251.

Matsuda, N. (1999). Wave model and GA to support kanseic evaluation: An application

to AHP. A paper presented at the 2nd Japan-Korean Joint Conference on Kansei

Engineering. Kong'ju, Korea.

Matsuda, N. (1999). Iterative use of flexible AHP as an aid to impression formation and decision making. In Osawa, M. (Ed.) Toward Impression Engineering. Tokyo: Maruzen Planets.

Matsuda, N. (1997). Kansei Information Design. Tokyo: Ohm.

Saaty, T.L. (1980). The Analytic Hierarchical Process. New York: McGraw-Hill. 